\title{
A Mobility Improvement Handover Scheme for Mobile-WiMAX
}

\author{
Md. Imtiyaz Anwar \\ National Institute of \\ Technology Jalandhar (India)
}

\author{
Arun Khosla \\ National Institute of \\ Technology Jalandhar (India)
}

\author{
Neetu Sood \\ National Institute of \\ Technology Jalandhar (India)
}

\begin{abstract}
This paper presents a mobility improvement handover algorithm with less scan time implementation for Mobile WiMAX. Mobile WiMAX is a wireless technology based on IEEE802.16e for broadband wireless access. Mobile WiMAX introduces the most significant new feature, mobility to support for handovers, which can be considered as a basic requirement for mobile communication system. The mandatory handoff method is Hard Handoff for Mobile WiMAX and other two optional soft handoff methods are Macro Diversity Handoff and Fast Base Station Switching Handoff method. Handover delay generates during data transmission but handover delay should be less than 50milli second for real time applications such as VoIP. The existing draft standard considers only the received signal strength (RSS) when deciding handover. The mobility improvement handover scheme that depends upon the velocity factor has been taken into consideration in this paper. The proposed scheme skips some unnecessary handover stages, reduces handover delay by adjusting the handover parameters viz. handover RSS trigger and threshold handover RSS trigger value to improve the velocity of the mobile station. The proposed scenario has been implemented using QualNet network simulator which has lead to less handover time.
\end{abstract}

Index Terms- Mobile WiMAX, Handover, Delay, Mobility, RSS Trigger, Velocity, Scanning trigger

\section{INTRODUCTION}

In order to achieve the high data rate in wireless services such as VoIP and IPTV, the Mobile WiMAX (Worldwide Interoperability for Microwave Access) based on the IEEE 802.16e [1] standard is developed as broadband wireless solution to the wired backhaul. Fixed WiMAX, based on IEEE 802.16 standard is a cost effective fixed wireless alternative to cable and DSL services. IEEE 802.16e is the new version to the Fixed WiMAX [2] adds mobility as a new feature and thus WiMAX provides high data rate mobile wireless services for metropolitan areas. WiMAX coverage range is up to thirty mile radius and data rates between 1.5 Mbps to 75 Mbps theoretically.

Handover (HO) is one of the key requirements to embrace mobility and Quality of Service (QoS) for the subscribers in IEEE 802.16e. HO refers to the process in which an MS migrates from the air interface provided by one BS to an air interface provided by another BS. The IEEE 802.16e defines three basic types of $\mathrm{HO}$ [3]: Hard Handover (HHO), Macro Diversity Handover (MDHO) and Fast Base Station Switching (FBSS). MDHO and FBSS are soft handovers and adopt the make-before-break scheme. HHO is mandatory in WiMAX system but MDHO and FBSS are optional. In HHO, the MS is connected to only one BS at a time. If the MS decides to connect to the new target BS, it selects only one target BS from a group of BSs and MS stops its radio link with the serving BS before establishing its radio link with the target BS. HHO is simple but causes long HO delay when velocity of MS is high. In MDHO or FBSS scheme, a MS is registered to several BSs at the same time. For MDHO, a MS communicates with two or more BSs in a given interval, while for FBSS, service flows are set up with multiple BSs. All discussions and analysis are based on hard handover.

A few papers have proposed several schemes to deal with research about HO in IEEE 802.16e.Some researchers used soft handover and others used hard handover schemes. In [4], velocity adaptive handover scheme is used but $\mathrm{HO}$ delay is below 50ms limit until the MS's velocity is up to 100.8 $\mathrm{Km} / \mathrm{h}$. This paper not included the scanning time reduction.

A mobility improvement handover scheme for Mobile WiMAX is proposed here to reduce the HO delay and the waste of wireless network resource with greater speed of the MS. Received Signal Strength Indicator (RSSI) is typically used as a measure of signal quality in WiMAX system. So, RSSI and MS's mobility are the most important factors to initiate HO. Apart from this, scanning time should also be reduced to minimize $\mathrm{HO}$ delay. In the proposed scheme, threshold HO value is varying with MS's velocity and minimized the time duration between RSS scan and threshold $\mathrm{HO}$ to reduce $\mathrm{HO}$ delay.

The remainder of this paper is organized as follows: Section 2 describes the HO process in Mobile WiMAX. Section 3 proposes mobility improvement HO scheme and performance analysis of the proposed $\mathrm{HO}$ scheme are presented in the next section. Finally the conclusion is mentioned in the last section.

\section{HANDOVER PROCESS}

The MAC (Medium Access Control) layer HO process of Mobile WiMAX consists of two phases. Network Topology Acquisition (NTA) is the first phase which is carried out 
before the actual $\mathrm{HO}$ process. The actual $\mathrm{HO}$ process including $\mathrm{HO}$ decision, initiation, ranging and network reentry process is performed. This paper contains only brief explanation of each $\mathrm{HO}$ phase as follows.

\section{A. Network Topology Acquisition (NTA)}

Network Topology Acquisition process contains Network Topology Advertisement, MS scanning of neighbour BSs and association process before $\mathrm{HO}$ procedure as shown in fig. 1 . The BS periodically sends an advertisement message, MOB_NBR_ADV, which includes channel information of the neighbouring BSs such as BS ID (Identifier), radiation power, frequency assignment, mobility and handover support and their UCD (Uplink Channel Descriptor) and DCD (Downlink Channel descriptor) information. The information contained in the MOB_NBR_ADV message processes faster handover to one BS out of all neighbouring BSs. After synchronizing with the neighbouring BSs, scanning of neighbouring BSs can be started. The MOB_SCAN_REQ/RSP message contains the information about scanning procedure with the serving BS. All downlink and uplink transmissions are paused during scanning process and the next step, the association procedure optionally perform between MS and the neighbouring BSs. Initial ranging process performed during MS's scanning interval is optional. The association procedure is to enable the MS to set and record ranging parameters and other information. By reusing those information MS is able for future $\mathrm{HO}$ to a target BS. If the MS decides to skip the association process, it must perform an initial ranging procedure with the target BS.

\section{B. Handover Process}

HO process refers that When MS migrates from the serving BS to the target BS

This step performs the actual handover to a new target BS when MS migrates from the serving BS. After NTA, MS can start cell reselection in order to select the target BS. If it refers the same operation with NTA, this stage can be abbreviated. After cell reselection, HO decision and Initiation is performed. Ms as well as BS can initiate $\mathrm{HO}$ decision and initiation process using MOB_MSHO_REQ/ MOB_BSHO_REQ message.

However, there exists another ambiguity for time of $\mathrm{HO}$ initiation. After target BS is decided for HO, MS sends MOB_HO-IND message to the serving BS and actual HO process begins. If the $\mathrm{HO}$ notification is transferred through backbone connection by the target BS, non contention based initial ranging can be assigned, which minimizes the $\mathrm{HO}$ delay.

If all physical parameters are adjusted, the network reentry process is initiated. Details of association level and security support are not included in this paper. To shorten the HO delay and to reduce wireless channel resource waste, a good handover scheme should be present. But in spite of that, some factors degrading the system performance also exist in the HO process. Suppose that a MS moves with low velocity, the network topology architecture may maintain the same in a long time, thus in the cell reselection stage, the MS can use the same network topology information and skip this stage. Since the channel information of the neighbouring BSs do

\section{NTA before HO}

-Network Topology Advertisement

-MS scanning of neighbour BSs

-Association Process

\section{HO Process during HO}
-Cell reselection
-HO decision and Initiation
-Close all connection (break- before make)
-Synchronize with new downlink and obtain parameters
-Obtain uplink parameters
-Ranging and uplink parameter adjustment
-Re-register
-MS re-authorization
-Termination with the serving BS

\section{Fig. 1. HO Procedure}

not change, MS could synchronize to target BS downlink by performing non-contention based ranging and therefore, the HO delay minimizes. On the other hand, when the MS moves with high velocity, the rapid change of channel condition makes pre obtained information useless. So, during actual $\mathrm{HO}$ procedure, the neighbouring BSs scanning and contention based ranging operation must be performed, which generates a long handover delay and wireless channel resource waste at higher speed of the MS. To deal with these problems, a new mobility improvement handover scheme is presented in this paper with reduce scanning time considering velocity as important parameter.

\section{PROPOSED HANDOVER SCHEME}

In this part, the mobility improvement handover scheme in which threshold $\mathrm{HO}$ value greatly depend on velocity of the MS and scanning trigger value has been proposed. Specification of IEEE802.16e [5] defines the HO procedures, but don't give information about $\mathrm{HO}$ decision. In general, the MS makes the HO decision according to signal quality in WiMAX, which can be measured by the RSSI. A model for $\mathrm{HO}$ is shown (Fig. 2) in which two BSs are there and one MS is moving with a certain velocity from one BS (serving BS) towards other BS (target BS), HO takes place.

Threshold HO $\left(T h_{\text {handover }}\right)$ represents the point for handover and threshold drop ( $\left.T h_{\text {drop }}\right)$ is the point below which the communication link becomes very weak and unacceptable. Below threshold drop point packet loss will be more and leads to excess delay of data transfer. Hysteresis margin, denoted by $\triangle H$, represents the difference between $T h_{\text {handover }}$ and $T h_{\text {drop }}$ point. The hysteresis value is used to 
eliminate the thrashing effect. The MS select those BSs whose RSSI value is higher than the serving BS which results in a better link for communication with the target BS with lower bit error rate (BER). Typically HO initiated when the RSSI of the serving BS is less than the $T h_{\text {handover }}$ and executed only if there is another BS having RSSI is at least $\triangle \mathrm{H}$ higher than the threshold drop.

$$
R S S I_{\text {target }}>T h_{\text {drop }}+\text { hysteresis value }
$$

The network channel information will change frequently when the MS's velocity is high, some unnecessary stages must be performed, which makes severe degradation in system performance such as $\mathrm{HO}$ delay and network resource waste. If the $T h_{\text {handover }}$ is set higher, the frequency of $\mathrm{HO}$ initiated will be higher, thus the network channel information can be acquired quickly so that the probability of preobtained information used in the $\mathrm{HO}$ process will be improved. It can reduce the HO delay but more waste of network channel resource. If the $T h_{\text {handover }}$ is set lower, frequency of $\mathrm{HO}$ will be lower, therefore $\mathrm{HO}$ delay will be longer but consumption of wireless channel resource will be less. But if $T h_{\text {handover }}$ has a constant value independent of the velocity, for a high $T h_{\text {handover }}$ the wireless channel resource waste will increase in the low velocity condition, and for a low threshold value, the HO delay will increase in the high velocity condition.

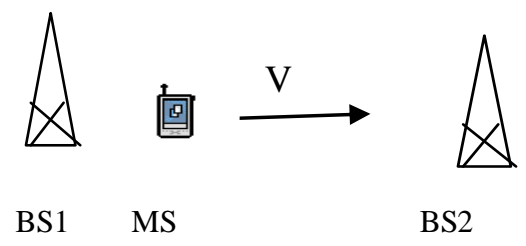

Figure 2. Proposed Model

To balance the more HO delay as well as the wireless channel resource waste problems, the mobility improvement handover scheme is proposed. The scheme changes the $T h_{\text {handover }}$ value according to velocity and minimization of scanning interval i.e the time interval between scanning trigger and $\mathrm{HO}$ trigger.

$$
T h_{\text {handover }}=T h_{\text {drop }}+\triangle H
$$

The description of analysis is not presented in this paper. $\triangle \mathrm{H}$ should be as low as possible for low velocity and for high velocity, $T h_{\text {handover }}$ have no effect on HO delay. So, $\triangle \mathrm{H}$ will maintain nearly a constant value at high velocity of the MS. In other situation, the hysteresis value would be larger at higher velocity. So, a factor is proposed which is associated with threshold value, speed and indirectly scanning time. After manipulating different equations [4], the HO threshold is given as below:

$$
T h_{\text {handover }}=T h_{\text {drop }}\left[1+\log _{2}(\mathrm{v}+1)\right]
$$

$T h_{\text {drop }}$ value can be varied with velocity, but here it is kept constant for simple simulation. Scanning trigger is also varied with velocity to reduce HO delay in our simulation process. In the next section performance analysis with results are discussed in which the mobility is improved and compared with other techniques.

\section{PERFORMANCE ANALYSIS}

For performance analysis of Mobile WiMAX, QualNet Network Simulator 4.5 is used. The HO threshold and scanning trigger value has an influence on HO delay, which depend on the velocity of the MS. Few main parameters for simulation purpose are listed in table 1.

Table 1. Simulation Parameters

\begin{tabular}{|l|l|}
\hline Simulation Parameters & Value \\
\hline Handover type & HHO \\
\hline Propagation model & Two-ray \\
\hline Default HO-RSS-TRIGGER & $4 \mathrm{~dB}$ \\
\hline Default HO-SCAN-TRIGGER & $5 \mathrm{~dB}$ \\
\hline Number of moving MS & 1 \\
\hline Number of BSs & 2 \\
\hline Channel Frequency & $2.4 \& 2.5 \mathrm{GHz}$ \\
\hline Drop threshold & $2 \mathrm{~dB}$ \\
\hline Step of simulation & $1 \mathrm{~m} / \mathrm{s}$ \\
\hline Speed range & $40 \mathrm{~m} / \mathrm{s}$ \\
\hline Constant bit rate & $1.2 \mathrm{Mbps}$ \\
\hline FFT size & 2048 \\
\hline
\end{tabular}

In the simulation process, the speed of MS is varied from $1 \mathrm{~m} / \mathrm{s}$ to $40 \mathrm{~m} / \mathrm{s}$ with $1 \mathrm{~m} / \mathrm{s}$ step. In simulation tool Two-ray model can only be selected for IEEE 802.16e.

In the constant threshold $\mathrm{HO}$ scheme, threshold value is constant (HO threshold $4 \mathrm{~dB}$, drop threshold $2 \mathrm{~dB}$, scan trigger) with variable velocity and the velocity adaptive $\mathrm{HO}$ scheme as well as the proposed scheme for HO adopts different threshold value according to the velocity variation. The simulation results and comparison between three different schemes are shown in Figure 3.

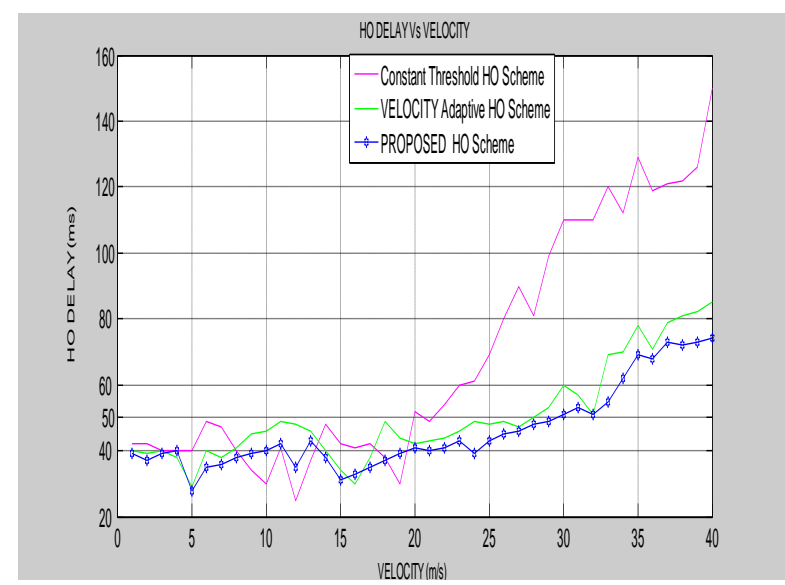

Figure 3. HO delay changes with velocity 
It is analyzed that when the MS moves with a low speed up to $20 \mathrm{~m} / \mathrm{s}$, there is little impact on the velocity adaptive $\mathrm{HO}$ scheme and the proposed scheme. But when the MS moves with a speed greater than $20 \mathrm{~m} / \mathrm{s}$, the HO delay is greatly reduced after implementing the proposed scheme. In constant threshold HO scheme, the HO delay remains below the $50 \mathrm{~ms}$ limit (Defined for Mobile WiMAX by WiMAX Forum) until the velocity rises up to $20 \mathrm{~m} / \mathrm{s}$ and the $\mathrm{HO}$ delay is growing and $150 \mathrm{~ms}$ at $40 \mathrm{~m} / \mathrm{s}$.

In velocity adaptive $\mathrm{HO}$ scheme the $\mathrm{HO}$ delay remains below the $50 \mathrm{~ms}$ limit until the velocity rises up $28 \mathrm{~m} / \mathrm{s}$ and $\mathrm{HO}$ delay is increasing up to $85 \mathrm{~ms}$ at $40 \mathrm{~m} / \mathrm{s}$ velocity.

In this proposed scheme, the $\mathrm{HO}$ delay is below the $50 \mathrm{~ms}$ limit up to $29 \mathrm{~m} / \mathrm{s}$ and at higher speed, the $\mathrm{HO}$ delay increases only up to $74 \mathrm{~ms}$ at $40 \mathrm{~m} / \mathrm{s}$. Reduction in $\mathrm{HO}$ delay indicates that some unnecessary consuming steps are not performed such as scanning duration In the proposed scheme, the HO delay reduced greatly as compared to both, constant threshold HO scheme and velocity adaptive HO scheme.

\section{CONCLUSION}

A mobility improvement $\mathrm{HO}$ scheme for IEEE 802.16e is implemented and analyzed here. According to the existing draft version of IEEE 802.16e standard, the HO will be initiated when the RSSI of the serving BS is lower than the threshold value. It does not consider the effect of velocity and scan threshold value, the HO threshold is set as a constant. But the velocity of the MS affects the HO delay. To cope with this problem, mobility improvement $\mathrm{HO}$ scheme is proposed, in which the HO threshold varied with velocity of the MS. The results show that the HO delay below 50ms limit when speed changes from $20 \mathrm{~m} / \mathrm{s}$ to $29 \mathrm{~m} / \mathrm{s}$ and the HO delay greatly reduced when the speed exceeds $29 \mathrm{~m} / \mathrm{s}$ which is approximately equal to $105 \mathrm{~km} / \mathrm{h}$. Thus, this HO scheme can provide seamless communication for Mobile WiMAX in high velocity and real time applications.

\section{REFERENCES}

[1] Caiyong HAO, Hongli LIU, Jie ZHAN, "A Velocity Adaptive Handover Scheme for Mobile WiMAX," Int. J. Communications, Network and System Sciences, Vol. 2, pp. 874-878, Dec. 2009.

[2] D. H. Lee and K. Kyamakya, "Fast handover algorithm for IEEE 802.16e broadband wireless access system,” IEEE Wireless Pervasive Computing Conference, pp. 16-18 January 2006.

[3] H. Fattah, H. Alnuweiri, "A New Handover Mechanism for IEEE8802.16e Wireless Networks," Wireless Communications and Mobile Computing Conference, August 2008.

[4] IEEE 802.16e/D12, "Air interface for fixed and mobile broadband wireless access systems: amendment for physical and medium access control layers for combined fixed and mobile operation in licensed bands," 2005.

[5] IEEE 802.16e-2005, IEEE Standard for local and metropolitan area networks-Part16: "Air interface for fixed and mobile broadband wireless access systems-amendent2: physical and medium access control layers for combined fixed and mobile operation in licensed band," 2006.

[6] IEEE Std 802.16-2004 (Revision of IEEE Std 802.16- 2001), "IEEE standard for local and metropolitan area networks-Part16: Air interface for fixed and mobile broadband wireless access systems," 2004.

[7] Kyung-ah K, Chong-Kwon K, Tongsok K, "A seamless handover Mechanism for IEEE 802.16e Broadband Wireless Access", August 2004.

[8] WiMAX Forum, Mobile WiMAX-Part I: "A technical overview and performance evaluation," 2006. 